\title{
INTERVALO HÍDRICO ÓTIMO PARA AVALIAÇÃO DA DEGRADAÇÃO FÍSICA DO SOLO ${ }^{(1)}$
}

\author{
Rachel Muylaert Locks Guimarães ${ }^{(2)}$, Cássio Antonio Tormena ${ }^{(3)}$, Éverton Blainski $^{(4)}$ \& \\ Jonez Fidalski ${ }^{(5)}$
}

\section{RESUMO}

A influência do sistema de uso e manejo na qualidade física do solo tem sido tema de destaque em razão de seus impactos ambientais e agronômicos. $O$ intervalo hídrico ótimo (IHO) é um moderno indicador da qualidade física que potencialmente pode apresentar os mecanismos e processos de perda ou recuperação da qualidade física do solo, por causa do seu uso e manejo. Diante disso, o objetivo deste trabalho foi quantificar a influência de diferentes sistemas de uso e manejo no IHO de um Latossolo Vermelho distrófico, textura francoarenosa, composto por $170,40 \mathrm{e} 790 \mathrm{~g} \mathrm{~kg}^{-1} \mathrm{de}$ argila, silte e areia, respectivamente. Foram selecionadas quatro áreas, sob os seguintes sistemas de uso e manejo do solo: mata nativa; pastagem cultivada por mais de 20 anos; citros por mais de 10 anos, antecedido por 10 anos de pastagens; e cultivo com culturas comerciais (milho, sorgo, aveia e mandioca), por cerca de 15 anos, após 10 anos de pastagem cultivada. Foram coletadas 48 amostras indeformadas, em cada área, no centro da camada de 0-0,10 $\mathrm{m}$ de profundidade. As amostras foram submetidas a potenciais de -10 a $-15.000 \mathrm{hPa}$, em que foram determinadas a curva de retenção de água, a curva de resistência do solo à penetração e, posteriormente, a densidade do solo (Ds) e o IHO. A Ds foi influenciada pelos sistemas de uso e manejo do solo na sequência cultivo $=$ citros $>$ pastagem $>$ mata nativa. A relação entre IHO e Ds foi linear e negativa, à exceção da mata nativa que na faixa de Ds entre $1,35-1,55 \mathrm{Mg} \mathrm{m}^{-3}$ apresentou relação positiva. Valores de $\mathrm{Ds}$, em que o $\mathrm{IHO}=0$, associados com severa degradação física do solo, foram de 1,75 e $1,80 \mathrm{Mg} \mathrm{m}^{-3}$, nos solos sob citros e cultivo,

(1) Trabalho financiado pelo CNPq - processo 420.088/99-7. Recebido para publicação em 02 de agosto de 2012 e aprovado em 31 de julho de 2013.

(2) Professora Adjunta, Departamento de Agronomia, Universidade Tecnológica Federal do Paraná. Via do Conhecimento, km 1. CEP 85503-390 Pato Branco (PR). E-mail: rachelguimaraes@utfpr.edu.br

(3) Professor Associado, Departamento de Agronomia, Universidade Estadual de Maringá. Av. Colombo, 5790, Bloco J-45. CEP 87020-900 Maringá (PR). Bolsista do CNPq. E-mail: catormena@uem.br

(4) Pesquisador, Empresa de Pesquisa Agropecuária e Extensão Rural de Santa Catarina, Centro de Informações de Recursos Ambientais e de Hidrometeorologia de Santa Catarina (Epagri/CIRAM). Rodovia Admar Gonzaga, 1347, Itacorubí. Caixa Postal 502. CEP 88034-901 Florianópolis (SC). E-mail: evertonblainski@epagri.sc.gov.br

(5) Pesquisador, Instituto Agronômico do Paraná, Estação Experimental de Paranavaí. Caixa Postal 564. CEP 87701-970 Paranavaí (PR). Bolsista da Fundação Araucária. E-mail: fidalski@iapar.br 
respectivamente; a proporção desses valores foi de $21 \%$ para o solo sob citros e $18 \%$, para o sob cultivo. A perda de qualidade física do solo foi menos acentuada no solo sob pastagem, quando comparado com os solos sob citros e cultivo, sendo a diminuição do IHO menos acentuada com o aumento da Ds. O menor valor de Ds em que iniciou a redução do IHO foi tomado como densidade do solo de alerta $\left(\mathrm{Ds}_{\mathrm{a}}\right)$. A $\mathrm{Ds}_{\mathrm{a}}$ foi de $1,55 \mathrm{Mg} \mathrm{m}^{-3}$ e pode ser utilizada como valor de referência no processo de recuperação da qualidade física do solo, válida para solos com textura, uso e manejo semelhante ao deste estudo.

Termos de indexação: densidade do solo, estrutura do solo, resistência do solo à penetração, água no solo, qualidade física do solo.

\title{
SUMMARY: LEAST LIMITING WATER RANGE FOR THE ASSESSMENT OF SOIL PHYSICAL DEGRADATION
}

\begin{abstract}
The effects of soil use and management on soil physical quality have been largely discussed due to its environmental and agronomic impacts. The least limiting water range $(L L W R)$ is a modern indicator of soil physical quality that can indicate processes and mechanisms linked to soil physical degradation caused by soil use and management. The aim of this work was to quantify the influence of different land uses and soil tillage on the LLWR of an Oxisol (170, 40 and $790 \mathrm{~g} \mathrm{~kg}^{-1}$ clay, silt and sand). Four areas were selected: native forest; planted pasture for more than 20 years; area under citrus cultivation for more than 10 years; area with annual crops (corn, sorghum, oat, and cassava) for 15 years after 10 years of pasture. In each treatment, 48 undisturbed samples were collected in the 0-0.10 m layer. A matric potential of -10 to -15,000 hPa was applied and the soil samples were analyzed for soil bulk density (Bd), water retention curve, soil resistance curve, $L L W R$, and critical Bd. Bulk density was influenced by soil use and management at the following sequence commercial crops $=$ citrus $>$ pasture $>$ native forest. The relationship between LLWR and Bd was linear and negative, except under native forest, where the $B d$ range (1.35-1.55 $\left.\mathrm{Mg} \mathrm{m}^{-3}\right)$ had a positive relationship. In the areas under citrus and annual crops, LLWR reached zero, with a critical bulk density $\left(B d_{c}\right)$ of 1.75 and $1.80 \mathrm{Mg} \mathrm{m}^{-3}$, respectively, with severe soil physical degradation. The ratio of these values was $21 \%$ for soil under citrus and $18 \%$ for soil under commercial crops. The loss of soil physical quality was less marked for the soil under pasture than citrus and annual crops, with a less pronounced decrease of LLWR with increasing $B d$. The lowest Bd value, at which the reduction of LLWR begins, was named alert bulk density $\left(B d_{a}\right)$. The $B d_{a}$ was $1.55 \mathrm{Mg} \mathrm{m}^{-3}$ and can be used as a reference value for the process of soil regeneration, valid for soils with soil texture and soil use and management similar to this study.
\end{abstract}

Index terms: soil bulk density, soil structure, soil resistance to penetration, soil water, soil physical quality.

\section{INTRODUÇÃO}

A degradação física do solo é um dos componentes do processo de sua degradação, geralmente associado às perdas de matéria orgânica e da integridade estrutural dos solos. Como consequência dessa degradação, ocorrem alterações em propriedades do solo que controlam os processos fundamentais relacionados com a dinâmica de água, ar e calor (Topp et al., 1994; Feddema, 1998) e crescimento das raízes. Assim, a quantificação dos impactos do manejo sobre a qualidade física do solo deveria ser realizada por meio de atributos que determinam os processos físicos que interferem em aspectos fisiológicos ligados ao crescimento e desenvolvimento das plantas.

A qualidade física do solo é um termo relacionado com sua estrutura, e os danos causados pelo uso e manejo, como erosão, desertificação e suscetibilidade à compactação, são originários da sua degradação (Hubert et al., 2007). De acordo com Omuto (2008), a perda da qualidade física do solo é um processo gradual que se inicia com a deterioração da estrutura do solo e termina com a perda diferencial de partículas por meio da erosão. Em geral, a perda da qualidade física do solo está associada com modificações dos arranjos de agregados e poros do solo, reduzindo a disponibilidade de ar, água e nutrientes necessários à produção de biomassa (Six et al., 2004; Omuto, 2008). Essas mudanças interferem nas características da superfície do solo, resultando na compactação do solo (Lanzanova et al., 2007); nas mudanças na distribuição e no tamanho de poros (Dexter, 2004); na diminuição da taxa de infiltração de água no solo (Sobrinho et al., 2003); na diminuição da água disponível (Dexter, 2004); na perda de matéria orgânica (Álvaro-Fuentes et al., 2008); na redução da diversidade e atividade de microrganismos (Six et al., 2004); e no aumento da 
suscetibilidade à erosão do solo (Feddema, 1998; Lal, 2000; Richard et al., 2001).

Para avaliar os impactos que as práticas de manejo causam ao solo, é necessário, também, identificar as modificações em indicadores da qualidade física e estrutural desse. Essas modificações dificilmente podem ser quantificadas de maneira exata por métodos visuais (Omuto, 2008; Guimarães et al., 2011, 2013), além das dificuldades em estabelecer quais propriedades são mais influenciadas. Experimentos em longo prazo seriam necessários para identificar essas mudanças. Alternativamente, estudos podem comparar as propriedades físicas de solos cultivados e sob vegetação nativa, respeitando critérios pedológicos e geológicos, como argumentam Araújo et al. (2004). Há reconhecimento de que estudos de aspectos relacionados à disponibilidade de água e à estrutura do solo são fundamentais para o entendimento de muitos processos de interesse agrícola (Dexter \& Youngs, 1992; Hadas, 1997), necessários para o manejo do solo em um contexto agronômico, econômico e sustentável (Lal, 1994; Lal \& Stewart, 1994).

As propriedades físicas comumente utilizadas para caracterizar a condição física do solo são a densidade do solo (Ds) e distribuição de poros (Reynolds et al., 2008); a retenção e disponibilidade de água (Tormena et al., 2004); a resistência tênsil de agregados (Tormena et al., 2008; Guimarães et al., 2009); o índice S (Dexter, 2004); e o intervalo hídrico ótimo (IHO) (Silva et al., 1994; Tormena et al., 1998; Cavalieri et al., 2004; Blainski et al., 2009). Muitos autores relataram que não há uma única medida indicadora da qualidade física do solo, porém a integração entre alguns indicadores pode ser útil na sua identificação (Dexter, 2004). Nesse contexto, o IHO caracteriza-se por ser indicador da qualidade do solo, por integrar suas propriedades físicas essenciais para o crescimento e desenvolvimento das plantas, com a vantagem de relacionar as limitações desse, diretamente relacionadas com os fatores de resposta das culturas (Silva et al., 1994; Tormena et al., 1998).

O IHO incorpora informações úteis na identificação de processos ligados às mudanças na estrutura do solo (Klein \& Camara, 2007), tornando-se capaz de identificar a ocorrência da degradação física do solo, capaz de interferir negativamente no crescimento de plantas. Por definição, o IHO integra três fatores associados ao crescimento de plantas em função da Ds e, dessa forma, estabelece-se como indicador de qualidade estrutural do solo para produção de plantas (Silva et al., 1994; Topp et al., 1994; Silva \& Kay, 1997; Zou et al., 2000). As avaliações em diversos solos indicam que o IHO decresce acentuadamente com o aumento da Ds até valores de $\mathrm{IHO}=0$. Esse valor de Ds é denominado de densidade do solo crítica $\left(\mathrm{Ds}_{\mathrm{c}}\right)$ (Silva et al., 1994; Imhoff et al., 2001). Quando $\mathrm{Ds}_{\mathrm{s}}>\mathrm{Ds}_{\mathrm{c}}$, assume-se uma severa degradação física do solo, o que torna restrito o crescimento das plantas (Collares et al., 2008). Não é necessário que o IHO assuma a nulidade para indicar a degradação física do solo, uma vez que com a redução do IHO, em razão do aumento da Ds, a variação temporal e a espacial do conteúdo de água do solo $(\theta)$ podem resultar em estresses físicos às plantas, seja por restrição de aeração, quando $\theta$ é elevado, ou por causa da excessiva resistência do solo à penetração, em razão do secamento do solo (Bengough et al., 2006; Tormena et al., 2007; Blainski et al., 2012). Dessa forma, o IHO destaca-se como um moderno e importante indicador de qualidade física do solo (Silva et al.,1994; Imhoff et al., 2001; Leão et al., 2004; Tormena et al., 1999, 2007), utilizado em diferentes solos e sistema de uso e manejo.

Neste trabalho, propôs-se que, por meio do IHO, além da $\mathrm{Ds}_{\mathrm{c}}$, pode-se identificar a densidade do solo de alerta $\left(\mathrm{Ds}_{\mathrm{a}}\right)$, que corresponde ao valor de $\mathrm{Ds}$ em que os limites de água disponível do solo (AD) passam a ser definidos pela excessiva resistência do solo à penetração $(\mathrm{RP})(2,0 \mathrm{MPa})$ e, ou, pela reduzida porosidade de aeração (PA). A D $s_{a}$ caracteriza a fase inicial do processo de degradação física do solo, uma vez que o IHO torna-se menor do que a água disponível e a sua identificação torna-se importante para a adoção de medidas eficientes de recuperação e, ou, conservação dos solos agrícolas. O conhecimento da $\mathrm{Ds}_{\mathrm{a}}$ é importante no controle da degradação física do solo por permitir a antecipação de medidas de controle, evitando-se severas condições restritivas às plantas quando a $\mathrm{Ds}_{\mathrm{s}}>\mathrm{Ds}_{\mathrm{c}}$.

A partir do conhecimento da $\mathrm{Ds}_{\mathrm{a}}$ e $\mathrm{Ds}_{\mathrm{c}}$, o monitoramento da qualidade física do solo pode ser feito de forma simples e com custo reduzido por meio de medições de Ds. Leonel et al. (2007) concluíram que o valor de $\operatorname{Ds}_{\mathrm{c}}\left(1,40 \mathrm{Mg} \mathrm{m}^{-3}\right)$ foi muito próximo daquele em que a produção da cultura decresceu $\left(1,32 \mathrm{Mg} \mathrm{m}^{-3}\right)$ em um solo de textura média $(45 \%$ de argila). Collares et al. (2006) utilizaram de forma efetiva a $\mathrm{Ds}_{\mathrm{c}}$ para o monitoramento e controle da qualidade física do solo, indicando que o aumento na frequência de $\mathrm{Ds}_{\mathrm{s}}>\mathrm{Ds}_{\mathrm{c}}$ no perfil do solo compactado determinou severas reduções de produtividade na cultura do feijoeiro. Ressalta-se que a Ds é a propriedade física mais facilmente obtida na maioria dos estudos e exige aparato instrumental simples, barato e de fácil operação.

Nesse contexto, os objetivos deste trabalho foram determinar o IHO para um Latossolo Vermelho distrófico, sob diferentes sistemas de uso e manejo; quantificar a influência dos sistemas de uso e manejo do solo, após longo período de implantação, na qualidade física do solo, utilizando o IHO como indicador; e estabelecer os valores de $\mathrm{Ds}_{\mathrm{a}}$ e $\mathrm{Ds}_{\mathrm{c}}$, com fins de avaliação e monitoramento da condição física do solo.

\section{MATERIAL E MÉTODOS}

A amostragem do solo foi realizada em áreas agrícolas do município de Paranavaí, região noroeste do Estado do Paraná, em um Latossolo Vermelho 
distrófico (Embrapa, 2006), textura Franco-arenosa, composto por 170,40 e $790 \mathrm{~g} \mathrm{~kg}^{-1}$ de argila, silte e areia, respectivamente, desenvolvido a partir do arenito da Formação Caiuá. Foram selecionadas quatro áreas para a amostragem, de acordo com o histórico de uso e manejo do solo: mata nativa - floresta estacional semidecidual; solo sob pastagem - Brachiaria humidicola em pastejo extensivo de bovinos por mais de 20 anos, com lotação média de 1,5 UA ha-1 (1 UA = 1 animal com peso vivo de $450 \mathrm{~kg}$ ), sendo o uso anterior com a cultura do café e algodão; citros - solo sob pomar de laranja por mais de 10 anos, em sistema de manejo com herbicidas para o controle das plantas daninhas nas linhas das plantas e roçadas mecânicas nas entrelinhas; e cultivo - solo sob cultivo de mandioca e culturas anuais (milho, sorgo e aveia) por mais de 15 anos, tendo sido ocupado anteriormente por pastagens desde 1975. No momento da amostragem, a área estava cultivada com a cultura da mandioca na fase de colheita, sob preparo convencional do solo com aração e gradagem.

Em cada área selecionada, foram coletadas 48 amostras indeformadas de solo, aleatoriamente nas áreas de amostragem, no centro da camada de $0-10 \mathrm{~cm}$, com o auxílio de anéis de aço inox de $5 \mathrm{~cm}$ de altura e de diâmetro. As amostras, após preparadas no laboratório, foram saturadas por meio da manutenção de uma lâmina de água máxima de dois terços da altura dos anéis por 48 h. Nessas amostras, determinaramse a curva de retenção de água no solo (CRA), a curva de resistência do solo à penetração (CRS) e a Ds.

Para a determinação da CRA, adotou-se procedimento descrito em Silva et al. (1994) e largamente utilizado em estudos dessa natureza. Do total de 192 amostras (48 por sistema de manejo), essas foram divididas em 12 grupos de potencial mátrico distintos. Cada grupo conteve 16 amostras, sendo quatro amostras para cada sistema de manejo. Em cada grupo de amostras, foram aplicados os seguintes potenciais matriciais $(\psi):-10,-20,-40,-60,-80$ e $-100 \mathrm{hPa}$ em uma mesa de tensão, $-300,-500,-700,-5.000,-10.000$ e - $15.000 \mathrm{hPa}$, por meio de pressões aplicadas em placas porosas, conforme Dane \& Hopmans (2002). Após atingir o equilíbrio, as amostras foram utilizadas para determinar a resistência à penetração $(\mathrm{RP})$ e, em seguida, foram secas em estufa a $105{ }^{\circ} \mathrm{C}$ para determinação de $\theta$ e da Ds, conforme Blake \& Hartge (1986). Para obtenção das medidas de RP, utilizou-se de um penetrômetro eletrônico similar ao descrito por Tormena et al. (2008).

Para a definição do IHO, a CRA e CRS foram descritas matematicamente para cada sistema de uso e manejo. A CRA, expressa pela relação entre $\psi$ e $\theta$, foi ajustada utilizando a equação proposta por van Genuchten (1980), descrita na equação 1, adotando a restrição para $m=1-1 / n$. (Mualem, 1976):

$$
\theta=\frac{(\theta \mathrm{s}-\theta \mathrm{r})}{1+\left[(\alpha \cdot \psi)^{\mathrm{n}}\right]^{\mathrm{m}}}
$$

em que $\theta$ é o conteúdo de água do solo $\left(\mathrm{m}^{3} \mathrm{~m}^{-3}\right) ; \theta \mathrm{r}$, o conteúdo de água residual $\left(\mathrm{m}^{3} \mathrm{~m}^{-3}\right) ; \theta \mathrm{s}$, o conteúdo de água do solo saturado $\left(\mathrm{m}^{3} \mathrm{~m}^{-3}\right) ; \psi$, o potencial matricial; e $\alpha$ e $n$ são parâmetros do modelo. Uma adaptação foi feita ao modelo e a Ds foi incorporada no parâmetro $n$ na rotina de cálculo. Os coeficientes do modelo $\alpha, n, \theta$ s e $\theta$ r foram determinados iterativamente no ajuste do modelo aos dados, utilizando-se a rotina PROC NLIN do SAS (SAS, 1999).

A CRS, descrita pela relação funcional entre RP, Ds e $\theta$, foi obtida pelo ajuste dos dados ao modelo não linear proposto por Busscher (1990), equação 2, individualmente para cada sistema de uso e manejo:

$$
\mathrm{RP}=a \operatorname{Ds}^{b} \theta^{c}
$$

em que RP é a resistência do solo à penetração $(\mathrm{MPa})$; $\theta$, o conteúdo de água do solo $\left(\mathrm{m}^{3} \mathrm{~m}^{-3}\right) ; \mathrm{Ds}$, a densidade do solo ( $\left.\mathrm{Mg} \mathrm{m}^{-3}\right)$; e $a, b$ e $c$ são os coeficientes do modelo. A equação foi linearizada e o ajuste do modelo da CRS foi feito por meio da rotina PROC REG, empregando o programa estatístico SAS (SAS, 1999).

O IHO foi determinado, conforme Tormena et al. (1998). Os valores críticos de $\theta$ em que o crescimento das culturas foi influenciado pelo $\psi, \mathrm{RP}$ e PA foram obtidos na capacidade de campo $\left(\theta_{\mathrm{CC}}\right)$, ou $\theta$ no $\psi$ de $-100 \mathrm{hPa}$ (Reichardt, 1988); no ponto de murcha permanente $\left(\theta_{\mathrm{PMP}}\right)$, ou $\theta$ no $\psi$ de $-15.000 \mathrm{hPa}$ (Savage et al., 1996); na resistência à penetração $\left(\theta_{\mathrm{RP}}\right)$ de $2,0 \mathrm{MPa}$ (Taylor et al., 1966); e no $\theta$ em que a porosidade de aeração $\left(\theta_{\mathrm{PA}}\right)$ é de $0,10 \mathrm{~m}^{3} \mathrm{~m}^{-3}$ (Grable $\&$ Siemer, 1968). Os valores de $\theta_{\mathrm{CC}}$ e $\theta_{\mathrm{PMP}}$ foram obtidos nos potenciais de -100 e $-15.000 \mathrm{hPa}$, utilizando-se a CRA (Equação 1) ajustada aos dados experimentais. Os valores de $\theta$ em que a RP atinge 2,0 MPa foi obtido por meio da equação 2. Esse valor foi adotado em razão de diversos autores considerarem $\mathrm{RP}>2,0 \mathrm{MPa}$ como condição restritiva ao crescimento dos sistemas radiculares para lavouras anuais e pomares de citros (Silva et al., 1994; Tormena et al., 1998; Araújo et al., 2004; Blainski, et al., 2008). O valor de $\theta$ em que a porosidade de aeração foi de $0,10 \mathrm{~m}^{3} \mathrm{~m}^{-3}$ foi obtido a partir da expressão: [(1 - Ds/Dp) - 0,1], em que Dp é a densidade das partículas $\left(2,65 \mathrm{Mg} \mathrm{m}^{-3}\right)$. O IHO foi calculado como a diferença entre os limites superior e inferior de $\theta$, em que ocorrem os limites das propriedades físicas envolvidas. O limite superior é o menor valor de $\theta$, considerando $\theta_{\mathrm{CC}}$ ou $\theta_{\mathrm{PA}}$, e o inferior é o maior valor de $\theta$ entre $\theta_{\mathrm{PMP}}$ e $\theta_{\mathrm{RP}}$. Assim, a expressão do cálculo depende dos valores limites:

$$
\begin{aligned}
& \mathrm{IHO}=\theta_{\mathrm{CC}}-\theta_{\mathrm{PMP}}, \text { para } \theta_{\mathrm{CC}}<\theta_{\mathrm{PA}} \text { e } \theta_{\mathrm{PMP}}>\theta_{\mathrm{RP}} ; \\
& \mathrm{IHO}=\theta_{\mathrm{CC}}-\theta_{\mathrm{RP}}, \text { para } \theta_{\mathrm{CC}}<\theta_{\mathrm{PA}} \text { e } \theta_{\mathrm{PMP}}<\theta_{\mathrm{RP}}, \\
& \mathrm{IHO}=\theta_{\mathrm{PA}}-\theta_{\mathrm{PMP}} \text {, para } \theta_{\mathrm{CC}}>\theta_{\mathrm{PA}} \text { e } \theta_{\mathrm{PMP}}>\theta_{\mathrm{RP}}, \\
& \mathrm{IHO}=\theta_{\mathrm{PA}}-\theta_{\mathrm{RP}}, \text { para } \theta_{\mathrm{CC}}>\theta_{\mathrm{PA}} \text { e } \theta_{\mathrm{PMP}}<\theta_{\mathrm{RP}} .
\end{aligned}
$$

A densidade do solo de alerta $\left(\mathrm{Ds}_{\mathrm{a}}\right)$ foi determinada, considerando-se o menor valor de Ds em que a água disponível passa a ser restringida pela $\mathrm{RP} \mathrm{e}$, ou, $\mathrm{PA}$; 
a $\mathrm{Ds}_{\mathrm{c}}$ corresponde ao valor em que $\mathrm{IHO}=0 . \mathrm{A}$ comparação de médias da Ds foi feita, utilizando-se o intervalo de confiança, descrito por Gabriel (1978), usando o programa estatístico SAS (SAS, 1999).

\section{RESULTADOS E DISCUSSÃO}

A Ds foi igual para cultivo e citros, que foram superiores à pastagem e mata nativa (Figura 1). Os maiores valores de $D$ s foram verificados sob cultivo e citros, indicando os efeitos da compactação do solo nesses sistemas de manejo, em comparação com o sob pastagem e mata nativa. Esses resultados estão de acordo com Machado et al. (2008) e Blainski et al. (2008). Provavelmente, no solo sob pastagem, o sistema radicular das plantas contribuiu para a redução da Ds (Fidalski et al., 2007a), com valores intermediários entre mata e os solos sob cultivo. Nesse contexto, os resultados estão de acordo com Omuto (2008), em que em solo sob pastagem não houve indicação de degradação física do solo.

Os resultados do ajuste dos dados de RP ao modelo representado pela equação 2 para os diferentes sistemas de uso e manejo, encontram-se no quadro 1. Considerando o coeficiente de determinação $\left(\mathrm{R}^{2}\right)$, o modelo ajustado permitiu explicar entre 83 e $89 \%$ da variabilidade da $R P$, por meio de Ds e $\theta$. Todos os coeficientes do modelo foram altamente significativos pelo teste $\mathrm{t}(\leq 0,0009)$ e os resíduos apresentaram distribuição normal pelo teste de Shapiro-Wilk $(p<0,05)$. A RP variou positivamente com a Ds e

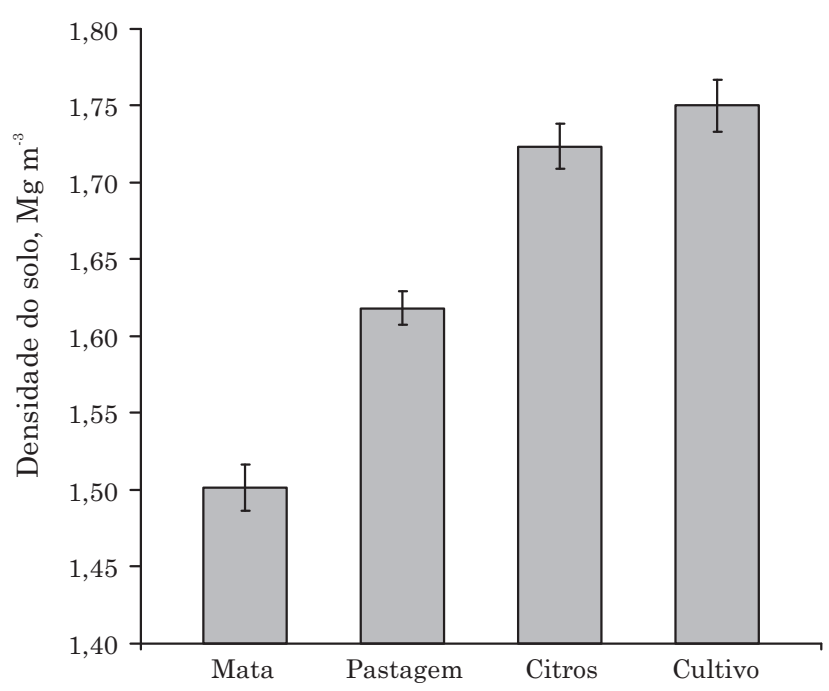

Figura 1. Valores médios de densidade do solo para cada tratamento e intervalo de confiança, indicados pelas barras verticais. Quando os limites superior e, ou, inferior do intervalo de confiança se sobrepõem não há diferença estatisticamente significativa entre os tratamentos $(\mathbf{p}<0,05)$. negativamente com $\theta$ (Quadro 1), em concordância com diversos trabalhos (Silva et al., 1994; Imhoff et al., 2000; Araújo et al., 2004; Cavalieri et al., 2009).

Os solos sob citros e cultivo apresentaram os maiores valores de $\mathrm{RP}$ evidenciados pelos valores dos coeficientes $b$ e $c$ (Quadro 1), ou seja, para um mesmo valor de Ds esses tratamentos evidenciaram maiores valores de RP, em comparação ao solo sob mata nativa. $\mathrm{O}$ maior valor atribuído ao coeficiente $b$ indicou que a RP foi fortemente influenciada pelo aumento da Ds. O aumento da RP em razão do aumento de Ds deveu-se ao efeito de fricção entre as partículas do solo; o aumento no teor de água exerceu efeito contrário por causa do efeito lubrificante da água que diminuiu o atrito ente as partículas do solo (Vepraskas, 1984).

Não foram constatados efeitos dos sistemas de uso e manejo do solo $(p<0,001)$ sobre a CRA. Dessa forma, ajustou-se apenas uma equação geral, utilizando os dados das quatro áreas de manejo (Equação 3). Apenas o parâmetro $n$ do modelo foi dependente da função linear da Ds (Equação 4). Todos os coeficientes do modelo de van Genuchten (1980) foram significativos, uma vez que o intervalo de confiança desses não incluiu o valor zero, conforme critério de Glantz \& Slinker (1990).

$$
\begin{gathered}
\theta=\left[0,0853+\left(\theta_{\mathrm{s}}-0,0853\right) /\left[(1+0,0518 \psi)^{n}\right]^{1-1 / n}\right] \\
n=3,7234-1,3102 \mathrm{Ds} \\
\mathrm{R}^{2}=\left[1-\left(\mathrm{SQ}_{\text {erro }} / \mathrm{SQ}_{\text {modelo }}\right)\right]=0,87
\end{gathered}
$$

Conforme o coeficiente de determinação $\left(\mathrm{R}^{2}\right)$ obtido, o modelo ajustado à CRA explicou $87 \%$ da variabilidade da retenção de água no solo. Resultados similares foram obtidos por Tormena \& Silva (2002) e Cavalieri et al. (2006). O ajuste do modelo aos dados experimentais não foi influenciado significativamente pelos sistemas de uso e manejo; o que se deve provavelmente às características texturais, pois a retenção de água do solo apresentou maior dependência da textura do que da estrutura na faixa de valores de $\psi$ mais baixos, próximos a $-15.000 \mathrm{hPa}$. Entretanto, a Ds interferiu na CRA, por influenciar a porosidade total e distribuição dos tamanhos de poros (Klein \& Libardi, 2002). O efeito significativo da Ds no parâmetro $n$ indicou que essa incorporou os efeitos do

Quadro 1. Modelos de regressão não linear da curva de resistência do solo à penetração para os diferentes tratamentos

\begin{tabular}{llc}
\hline & Equação de regressão(1) & $\mathbf{R}^{\mathbf{2}}$ \\
\hline Mata nativa & $\mathrm{RP}=0,0165 \mathrm{Ds}^{4,8411} \theta^{-1,1108}$ & 0,84 \\
Pastagem & $\mathrm{RP}=0,0175 \mathrm{Ds}^{5,2816} \theta^{-1,1710}$ & 0,83 \\
Citros & $\mathrm{RP}=0,0006 \mathrm{Ds}^{9,2205} \theta^{-1,8406}$ & 0,89 \\
Culturas anuais & $\mathrm{RP}=0,0006 \mathrm{Ds}^{8,7763} \theta^{-1,9048}$ & 0,88 \\
\hline
\end{tabular}

(1) Modelo: $\mathrm{RP}=\mathrm{a} \mathrm{Ds}^{\mathrm{b}} \theta^{\mathrm{c}} ; \theta \mathrm{s}$ : densidade do solo $\left(\mathrm{Mg} \mathrm{m}^{-3}\right) ; \theta$ : teor de água do solo $\left(\mathrm{m}^{3} \mathrm{~m}^{-3}\right)$ e RP: resistência do solo à penetração (MPa). 
uso e manejo do solo nas estimativas da retenção de água, por isso procedeu-se às estimativas de $\theta$ para cada sistema de uso e manejo do solo a partir da Ds obtida em cada uma das condições de uso e manejo avaliadas.

O IHO diminuiu em razão da intensificação do uso do solo, representado pela redução da área hachurada da figura 2. Essa intensificação proporcionou perdas da qualidade física e estrutural, evidenciada pela redução da amplitude do IHO, em ordem decrescente: mata, pastagem, citros e cultivo. Foi verificado efeito positivo da Ds sobre a retenção de água no solo, principalmente no potencial equivalente à capacidade de campo (Figura 2). $\mathrm{O}$ aumento da retenção de água do solo na capacidade de campo em todos os tratamentos e do ponto de murcha permanente no citros e cultivo está associado às modificações decorrentes da redução do diâmetro dos poros (Dexter et al., 2008). No $\psi=-15.000 \mathrm{hPa}$, o impacto da Ds sobre a retenção foi menor do que nos $\psi=-100 \mathrm{hPa}$ (capacidade de campo). Entretanto, o aumento da Ds implicou em maior massa de partículas com elevada superfície de adsorção por unidade de área, que teve principal efeito sobre a retenção de água em baixos potenciais.
A aeração não foi limitante para os solos sob mata nativa e pastagem (Figura 2), verificada por $\theta_{\mathrm{PA}}$ maior do que aquela equivalente a $\theta_{\mathrm{CC}}$. Nos solos sob citros e cultivo, os elevados valores de Ds implicaram em reduções nos macroporos, que favoreceram o rápido movimento de água sob elevados $\psi$, evidenciada por $\theta_{\mathrm{PA}}<\theta_{\mathrm{CC}}$ em valores de $\mathrm{Ds}>1,80 \mathrm{Mg} \mathrm{m}^{3}$.

O incremento da Ds, observado nos solos sob pastagem, citros e cultivo, teve grande impacto sobre $\theta_{\mathrm{RP}}$ (Figura 2). Observou-se que o maior valor de Ds obtido na mata nativa $\left(1,61 \mathrm{Mg} \mathrm{m}^{-3}\right)$ equivaleu aos menores valores de Ds nos demais tratamentos. Com exceção da mata nativa, $\theta_{\mathrm{RP}}$ foi considerada o limite inferior do IHO para toda a faixa de Ds estudada, (Figura 2). Isto implica em condições físicas menos favoráveis para o crescimento das plantas, por proporcionar restrições físicas a essas mesmo em condições em que o $\theta$ (ou $\psi$ ) não é limitante. Esse comportamento foi menos acentuado no solo sob pastagem (Figura 2), podendo estar associado ao maior teor de matéria orgânica do solo sob pastagem, à maior quantidade de raízes e à continuidade dos poros decorrentes da ausência de revolvimento do solo nesse sistema de uso. Todos esses fatores interferiram na vulnerabilidade do solo em aumentar a RP com o

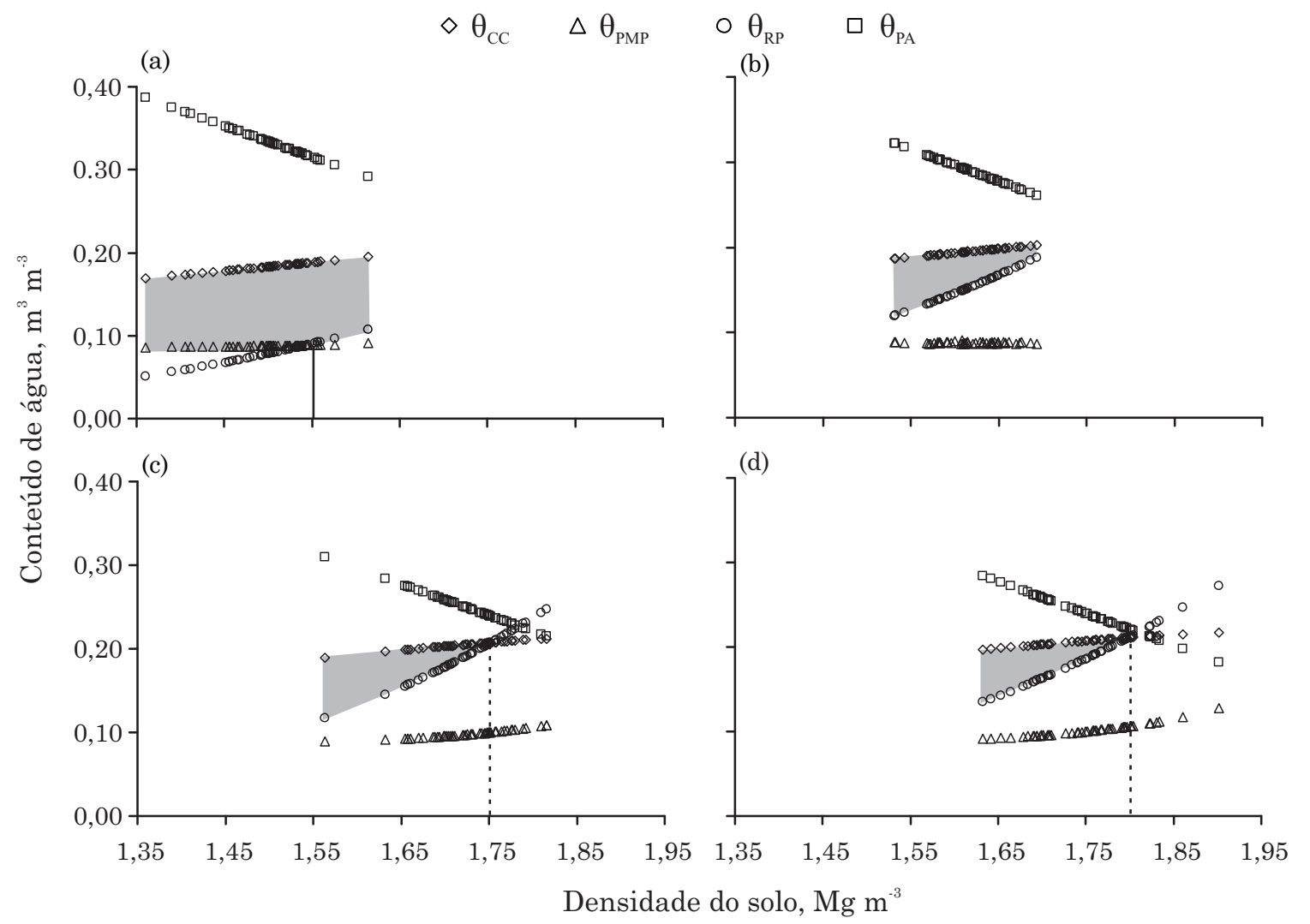

Figura 2. Variação do conteúdo de água $(\theta)$ em função da densidade do solo na capacidade de campo $\left(\theta_{\mathrm{CC}}=-100\right.$ $\mathrm{hPa})$, ponto de murcha permanente $\left(\theta_{\mathrm{PMP}}=-15.000 \mathrm{hPa}\right)$, porosidade de aeração $\left(\theta_{\mathrm{PA}}=0,10 \mathrm{~m}^{3} \mathrm{~m}^{-3}\right)$ e resistência do solo à penetração $\left(\theta_{R P}=2 \mathrm{MPa}\right)$ para os tratamentos mata nativa (a), pastagem (b), citros (c) e cultivo (d). A área hachurada indica o intervalo hídrico ótimo (IHO); as linhas pontilhadas, a $\mathrm{Ds}_{\mathrm{c}}(\mathrm{IHO}=0)$; e a linha sólida, $\mathrm{a} \mathrm{Ds}_{\mathrm{a}}(\mathrm{a})$. 
secamento, a exemplo do verificado por Fidalski \& Tormena (2007b).

A relação entre IHO e Ds foi linear e negativa, à exceção da mata nativa que na faixa de Ds entre 1,35 e $1,55 \mathrm{Mg} \mathrm{m}^{-3}$ apresentou relação positiva, revelando efeito benéfico do aumento do volume de poros de menor diâmetro na retenção de água (Figura 3). Nos solos sob mata nativa e pastagem, não foi identificada a ocorrência de $\mathrm{IHO}=0$, o que definiria um valor de $\mathrm{Ds}_{\mathrm{c}}$ (Figura 3). Na mata nativa, o limite superior do IHO foi determinado pela $\theta_{\mathrm{CC}}$, enquanto o inferior, pela $\theta_{\text {PMP para }} \mathrm{Ds}<1,55 \mathrm{Mg} \mathrm{m}^{-3}$. Esses resultados demonstraram que os menores valores de Ds registrados nesse tratamento ocasionaram menores restrições físicas dentro do intervalo de água disponível $\left(\theta_{\mathrm{CC}}-\theta_{\mathrm{PMP}}\right)$, em relação aos outros tratamentos. Outra inferência pode ser feita com relação ao coeficiente angular da relação IHO e Ds (Equações 5, 6, 7 e 8):

$$
\begin{array}{ll}
\text { Mata } & \text { IHO }=0,3598-0,1688 \mathrm{Ds} \\
\text { Pastagem } & \text { IHO }=0,5673-0,3250 \mathrm{Ds} \\
\text { Citros } & \text { IHO }=0,7188-0,4088 \mathrm{Ds} \\
\text { Cultivo } & \text { IHO }=0,6822-0,3781 \mathrm{Ds}
\end{array}
$$

sendo que a equação da reta para o solo sob a mata foi feita para a porção em que IHO diminui com a Ds.

O solo sob citros apresentou o maior coeficiente angular $(0,4088)$, revelando que com o aumento da Ds a redução do IHO foi mais acentuada do que nos demais tratamentos. Esses resultados indicaram que o solo sob citros apresentou maior degradação física, possivelmente em razão do manejo adotado. Esses valores sinalizam para maior vulnerabilidade do solo à degradação física, pois pequenas alterações de Ds podem acarretar grandes reduções na amplitude do IHO e consequentemente maior ocorrência de $\theta$ fora dos limites desse indicador de qualidade física do solo.

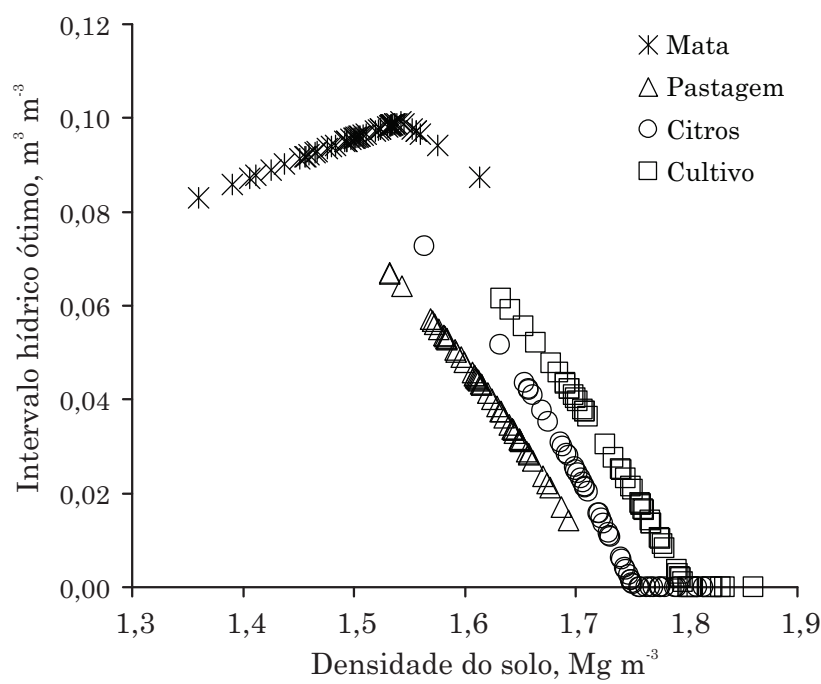

Figura 3. Variação do intervalo hídrico ótimo em função da densidade do solo.
No solo sob citros e cultivo, ocorreram valores de Ds em que IHO = 0 (Figura 3); nesses tratamentos, a $\mathrm{Ds}_{\mathrm{c}}$ foi de 1,75 e $1,80 \mathrm{Mg} \mathrm{m}^{-3}$, respectivamente (Figura 2), e a proporção de valores de $\mathrm{Ds}_{\mathrm{s}}>\mathrm{Ds}_{\mathrm{c}}$ sinalizou para a degradação física do solo. Tal proporção foi de $21 \%$ para o solo sob citros e $18 \%$, para o sob cultivo. A redução da proporção de $\mathrm{Ds}_{\mathrm{c}}$ no solo sob cultivo, em comparação com citros, pôde ser conferida pelo tráfego cumulativo de máquinas utilizadas no manejo do pomar. Práticas mecânicas devem ser adotadas nessas áreas para melhoria da qualidade física do solo em curto prazo e em seguida introduzidas culturas com sistema radicular agressivo para a área sob cultivo, visando o incremento do efeito residual da ação mecânica. Em pomares de citros, a adoção de cobertura permanente com gramíneas é opção para conter a degradação física, como indicaram os resultados de Fidalski et al. (2010).

$\mathrm{O}$ valor de $\mathrm{Ds}_{\mathrm{a}}$, definido pelo menor valor de $\mathrm{Ds}$ em que o intervalo de água disponível do solo começa a sofrer reduções, não pôde ser identificado claramente nos tratamentos com pastagem, citros e cultivo (Figura 2), pois a RP caracterizou o limite inferior do IHO em substituição ao PMP para toda faixa de valores de Ds observada. Esses resultados indicaram o elevado grau de degradação física do solo sob esses sistemas de uso e manejo. $\mathrm{O}$ valor de $\mathrm{Ds}_{\mathrm{a}}$ desse solo pode ser considerado como o maior valor de Ds observado na mata (Ds $=1,61 \mathrm{Mg} \mathrm{m}^{-3}$ ), apesar da discreta redução do IHO a partir de Ds $=1,55 \mathrm{Mg} \mathrm{m}^{-3}$ (Figura 2a). Portanto, valores entre 1,55 e $1,61 \mathrm{Mg} \mathrm{m}^{-3}$ devem ser o alvo de referência num processo de recuperação da qualidade física do solo em estudo.

A alteração do valor da $\mathrm{Ds}_{\mathrm{a}}$ para um valor menor do que o da mata nativa $\left(1,55 \mathrm{Mg} \mathrm{m}^{-3}\right)$, como verificado nos demais tratamentos, estendendo-se a curva de RP para baixo na figura 2, revelou a forte degradação física do solo ocasionada pelo uso e manejo do solo, especialmente no solo sob citros e sob cultivo. Em razão do uso e manejo do solo, o sistema poroso sofreu degradação (compactação), ocasionando alterações no arranjo dos poros, de forma que, mesmo na área com pastagem, o tempo de implantação de gramíneas não foi suficiente para recuperar a qualidade física do solo. No entanto, a melhor qualidade do solo na pastagem, evidenciada por Ds mais baixas quando comparadas aos solos sob citros e cultivo, demonstrou que, possivelmente, a implantação de um sistema de uso com pastagem, cujo manejo reduzisse o impacto do pisoteio e proporcionasse maior aporte de biomassa radicular e da parte aérea, contribuísse para a melhoria da qualidade do solo. Esses resultados indicaram perdas irrecuperáveis da estrutura e qualidade física do solo e que, em curto prazo, nem mesmo a reintrodução de florestas na área poderia também ser capaz de recuperar integralmente a estrutura do solo. Trabalhos nessa direção foram realizados por McHugh et al. (2009), evidenciando que o pleno restabelecimento a condições naturais do solo podem não acontecer em sistemas de uso com 
revolvimento do solo por causa do efeito adverso causado pelo manejo e tráfego de máquinas.

Portanto, medidas de controle da qualidade física do solo devem ser tomadas a partir dos valores de $\mathrm{Ds}_{\mathrm{a}} \mathrm{e}$ $\mathrm{Ds}_{\mathrm{c}}$, tendo como base o IHO. A recuperação do solo com vistas na $\mathrm{Ds}_{\mathrm{a}}$ é menos trabalhosa e de menor custo, podendo-se evitar condições de degradação física do solo severas e restritivas às plantas, como a ocorrência de valores de $\mathrm{Ds}_{\mathrm{s}}>\mathrm{Ds}_{\mathrm{c}}$. Apesar de o valor apresentado para $\mathrm{DS}_{\mathrm{a}}$ somente ser válido para o solo em estudo, o conceito da $\mathrm{Ds}_{\mathrm{a}}$ pode ser utilizado para outros, a partir da obtenção do IHO.

\section{CONCLUSÕES}

1. A redução do IHO foi mais acentuada no solo sob citros, revelando sua maior degradação sob esse uso e manejo, com o aumento da Ds.

2. Os solos sob citros e cultivo apresentaram IHO $=0$, sendo as proporções de amostras com $\mathrm{Ds}_{\mathrm{s}}>\mathrm{Ds}_{\mathrm{c}}$ de 21 e $18 \%$, para os sob citros e cultivo, respectivamente.

3. $\mathrm{ADs}_{\mathrm{a}}$ foi de $1,55 \mathrm{Mg} \mathrm{m}^{-3}$, revelada no solo sob mata nativa, e não pôde ser identificada claramente dentro da amplitude de Ds amostradas para os demais tratamentos, podendo, esse valor, ser utilizado como referência no processo de recuperação da qualidade física do solo.

\section{LITERATURA CITADA}

ÁLVARO-FUENTES, J.; ARRÚE, J.L.; GRACIA, R. \& LÓPEZ, M.V. Tillage and cropping intensification effects on soil aggregation: Temporal dynamics and controlling factors under semiarid conditions. Geoderma, 45:390-396, 2008.

ARAÚJO, M.A.; TORMENA, C.A. \& SILVA, A.P. Propriedades físicas de um Latossolo Vermelho distrófico cultivado e sob mata nativa. R. Bras. Ci. Solo, 28:337-345, 2004.

BENGOUGH, A.G.; BRANSBY, M.F.; HANS, J.; McKENNA, S.J.; ROBERTS, T. \& VALENTINE, T.A. Root responses to soil physical conditions: Growth dynamics from field to cell. J. Exp. Bot., 57:437-443, 2006.

BLAINSKI, E.; GONÇALVES, A.C.A.; TORMENA, C.; FOLEGATTI, M.V. \& GUIMARÃES, R.M.L. Intervalo hídrico ótimo num Nitossolo Vermelho distroférrico irrigado. R. Bras. Ci. Solo, 33:273-281, 2009.

BLAINSKI, E.; TORMENA, C.A.; GUIMARÃES, R.M.L. \& NANNI, M.R. Qualidade física de um Latossolo sob plantio direto influenciada pela cobertura do solo. R. Bras. Ci. Solo, 36:79-87, 2012.

BLAINSKI, E.; TORMENA, C.A.; FIDALSKI, J. \& GUIMARÃES, R.M.L. Quantificação da degradação física do solo por meio da curva de resistência do solo à penetração. R. Bras. Ci. Solo, 32:975-983, 2008.
BLAKE, G.R. \& HARTGE, K.H. Bulk density. In: KLUTE, A., ed. Methods of soil analysis: physical and mineralogical methods. 2.ed. Madison, American Society of Agronomy, 1986. p.363-375.

BUSSCHER, W.J. Adjustment of flat-tipped penetrometer resistance data to a common water content. Am. Soc. Agric. Eng., 33:519-524, 1990.

CAVALIERI, K.M.V.; TORMENA, C.A.; PINTRO, J.C.; COSTA, A.C.S.; FIDALSKI, J. \& SOUZA JUNIOR, I.G. Alterações nas propriedades químicas de um Latossolo. Acta Sci. Agron., 26:377-385, 2004.

CAVALIERI, K.M.V.; SILVA, A.P.; ARVIDSSON, J. \& TORMENA, C.A. Influência da carga mecânica de máquina sobre propriedades físicas de um Cambissolo Háplico. R. Bras. Ci. Solo, 33:477-485, 2009.

CAVALIERI, K.M.V.; TORMENA, C.A.; VIDIGAL FILHO, P.S.; GONÇALVES, A.C.A. \& COSTA, A.C.S. Efeitos de sistemas de preparo nas propriedades físicas de um Latossolo Vermelho distrófico. R. Bras. Ci. Solo, 30:137147, 2006.

COLLARES, G.L.; REINERT, D.J.; REICHERT, J.M. \& KAISER, D.R. Qualidade física do solo na produtividade da cultura do feijoeiro num Argissolo. Pesq. Agropec. Bras., 41:1663-1674, 2006.

COLLARES, G.L.; REINERT, D.J.; REICHERT, J.M. \& KAISER, D.R. Compactação de um Latossolo induzida pelo tráfego de máquinas e sua relação com o crescimento e produtividade de feijão e trigo. R. Bras. Ci. Solo, 32:933-942, 2008.

DANE, J.H. \& HOPMANS, J.W. Water retention and storage. In: DANE, J.H. \& TOPP, G.C., eds. Methods of soil analysis: Physical methods. Madison, American Society of America, 2002. Part 4. p.671-720.

DEXTER, A.R. \& YOUNGS, I.M. Soil physic toward 2000. Soil Till. Res., 24:101-106, 1992.

DEXTER, A.R. Soil physical quality. Part I - Theory, effects of soil texture, density, and organic matter, and effects on root growth. Geoderma, 120:201-214, 2004.

DEXTER, A.R.; CZYZ, E.A.; RICHARD, G. \& RESZKOWSKA, A. A user-friendly water retention function that takes account of the textural and structural pore spaces in soil. Geoderma, 143:243-253, 2008.

EMPRESA BRASILEIRA DE PESQUISA AGROPECUÁRIA . EMBRAPA. Sistema brasileiro de classificação de solos. 2.ed. Rio de Janeiro, 2006. 306p.

FEDDEMA, J.J. Estimated impacts of soil degradation on the African water balance and climate. Clim. Res., 10:127. $141,1998$.

FIDALSKI, J.; TORMENA, C.A. \& SILVA, A.P. Qualidade física do solo em pomar de laranjeira no Noroeste do Paraná com manejo da cobertura permanente na entrelinha. R. Bras. Ci. Solo, 31:423-433, 2007a.

FIDALSKI, J. \& TORMENA, C.A. Funções de pedotransferência para as curvas de retenção de água e de resistência do solo à penetração em sistemas de manejo com plantas de cobertura permanente em citros. Ci. Rural, 37:1316-1322, 2007b. 
FIDALSKI, J.; TORMENA, C.A. \& SILVA, A.P. Least limiting water range and physical quality of soil under groundcover management systems in citrus. Sci. Agric., 67:448-453, 2010.

GABRIEL, K.A. Simple method of multiple comparisons of means. J. Am. Statist. Assoc., 73:724-729, 1978.

GLANTZ, S.A. \& SLINTER, B.K. Primer of applied regression and analysis of variance. New York, McGraw-Hill, 1990. 777p.

GRABLE, A.R. \& SIEMER, E.G. Effects of bulk density, aggregate size, and soil water suction on oxygen diffusion, redox potential and elongation of corn roots. Soil Sci. Soc. Am. J., 32:180-186, 1968.

GUIMARÃES, R.M.L.; BALL, B.C. \& TORMENA, C.A. Improvements in the visual evaluation of soil structure. Soil Use Manage., 27:395-403, 2011.

GUIMARÃES, R.M.L.; BALL, B.C.; TORMENA, C.A.; GIAROLA, N.F.B. \& SILVA, A.P. Relating visual evaluation of soil structure to other physical properties in soils of contrasting texture and management. Soil Till. Res., 127:92-99, 2013.

GUIMARÃES, R.M.L.; TORMENA, C.A.; ALVES, S.J.; FIDALSKI, J. \& BLAINSKI, E. Tensile strength, friability and organic carbon in an Oxisol under a crop-livestock system. Sci. Agric., 66:499-505, 2009.

HADAS, A. Soil tilth - the desired soil structural state obtained through proper soil fragmentation and reorientation processes. Soil Till. Res., 43:7-40, 1997.

HUBERT, F.; HALLAIRE, V.; SARDINI, P.; CANER, L. \& HEDDADJ, D. Pore morphology changes under tillage and no-tillage practices. Geoderma, 142:226-236, 2007.

IMHOFF, S.; SILVA, A.P. \& TORMENA, C.A. Aplicação da curva de resistência do solo à penetração no controle da qualidade física de um solo sob pastagem. Pesq. Agropec. Bras., 35:1493-1500, 2000.

IMHOFF, S.; SILVA, A.P.; DIAS JÚNIOR, M.S. \& TORMENA, C.A. Quantificação de pressões críticas para o crescimento de plantas. R. Bras. Ci. Solo, 25:11-18, 2001.

KLEIN, V.A. \& CAMARA, R.K. Rendimento da soja e intervalo hídrico ótimo em Latossolo Vermelho sob plantio direto escarificado. R. Bras. Ci. Solo, 31:221-227, 2007.

KLEIN, V.A. \& LIBARDI, P.L. Densidade e distribuição do diâmetro dos poros de um Latossolo Vermelho sob diferentes sistemas de uso e manejo. R. Bras. Ci. Solo, 26:857-867, 2002.

LAL, R. \& STEWART, B.A., eds. Land degradation. Advances in soil science. New York, Springer, 1994. v.11.

LAL, R. Physical management of soils of the tropics: Priorities for the $21^{\text {st }}$ century. Soil Sci., 165:191-207, 2000.

LAL, R. Tillage effects on soil degradation, soil resilience, soil quality, and sustainability. Soil Till. Res., 27:1-8, 1994.

LANZANOVA, M.E.; NICOLOSO, R.S.; LOVATO, T.; ELTZ, F.L.F.; AMADO, T.J.C. \& REINERT, D.J. Atributos físicos do solo em sistema de integração lavoura-pecuária sob plantio direto. R. Bras. Ci. Solo, 31:1131-1140, 2007.
LEÃO, T.P.; SILVA, A.P.; MACEDO, M.C.M.; IMHOFF, S. \& EUCLIDES, V.P.B. Intervalo hídrico ótimo na avaliação de sistemas de pastejo contínuo e rotacionado. R. Bras. Ci. Solo, 28:415-423, 2004.

LEONEL, C.L.; CENTURION, M.A.P.C.; CENTURION, J.F.; BEUTLER, A.N. \& FREDDI, O.S. Relação da compactação do solo com a cultura do amendoim. Biosci. J., 23:70-81, 2007.

MACHADO, J.L.; TORMENA, C.A.; FIDALSKI, J. \& SCAPIM, C.A. Inter-relações entre as propriedades físicas e os coeficientes da curva de retenção de água de um Latossolo sob diferentes sistemas de uso. R. Bras. Ci. Solo, 32:495-502, 2008.

McHUGH; A.D.; TULLBERG, J.N. \& FREEBAIRN, D.M. Controlled traffic farming restores soil structure. Soil Till. Res., 104:164-172, 2009.

MUALEM, Y. A new model for predicting the hydraulic conductivity of unsaturated porous media. Water Res. Res., 12:513-522, 1976.

OMUTO, C.T. Assessment of soil physical degradation in Estern Kenya by use of a sequential soil testing protocol. Agric. Ecosyst. Environ., 128:199-211, 2008.

REICHARDT, K. Capacidade de campo. R. Bras. Ci. Solo, 12:211-216, 1988.

REYNOLDS, W.D.; DRURY, C.F.; YANG, X.M. \& TAN, C.S. Optimal soil physical quality inferred through structural regression and parameter interactions. Geoderma, 146:466-474, 2008.

RICHARD, G.; COUSIN, I.; SILLON, J.F.; BRUAND, A. \& GUÉRIF, J. Effect of compaction on the porosity of a silty soil: influence on unsaturated hydraulic properties. Eur. J. Soil Sci., 52:49-58, 2001.

SAVAGE, M.J.; RITCHIE, J.T.; BLAND, W.L. \& DUGAS, W.A. Lower limit of soil water availability. Agron. J., 88:844651, 1996.

SILVA, A.P. \& KAY, B.D. Estimating the least limiting water range of soil from properties and management. Soil Sci. Soc. Am. J., 61:877-883, 1997.

SILVA, A.P.; KAY, B.D. \& PERFECT, E. Characterization of the least limiting water range of soils. Soil Sci. Soc. Am. J., 58:1775-1781, 1994.

SIX, J.; BOSSUYT, H.; DEGRYZE, S. \& DENEF, K. A history of research on the link between (micro)aggregates, soil biota, and soil organic matter dynamics. Soil Till. Res., 79:7-31, 2004.

ALVES. SOBRINHO, T.; VITORINO, A.C.T.; SOUZA, L.C.F.; GONÇALVES, M.C. \& CARVALHO, D.F. Infiltração de água no solo em sistemas de plantio direto e convencional. R. Bras. Eng. Agric. Amb., 7:191-196, 2003.

STATISTICAL ANALYSIS SYSTEM INSTITUTE - SAS/STAT. Procedure guide for personal computers. Version 5. Cary, 1999.

TAYLOR, H.M.; ROBERSON, G.M. \& PARKER JR, J.J. Soil strength-root penetration relations to medium to coarsetextured soil materials. Soil Sci., 102:18-22, 1966.

TOPP, G.C.; GALGANOV, Y.T.; WIRES, K.C. \& CULLEY, J.L.B. Non-limiting water range (NLWR): An approach for assessing soil structure. Ottawa, Soil Quality Evaluation Program, Agriculture and Agr-Food Canada, 1994. 36p. (Technical Report, 2) 
TORMENA, C.A.; VIDIGAL FILHO, P.S.; GONÇALVES, A.C.A. \& ARAÚJO, M.A. Influência de diferentes sistemas de preparo do solo nas propriedades físicas de um Latossolo Vermelho distrófico. R. Bras. Eng. Agric. Amb., 8:65-71, 2004 .

TORMENA, C.A. \& SILVA, A.P. Incorporação da densidade do solo no ajuste de dois modelos à curva de retenção de água no solo. R. Bras. Ci. Solo, 26:305-314, 2002.

TORMENA, C.A.; ARAÚJO, M.A.; FIDALSKI, J. \& COSTA, J.M. Variação temporal do intervalo hídrico ótimo de um Latossolo Vermelho distroférrico sob sistemas de plantio direto R. Bras. Ci. Solo, 31:211-219, 2007.

TORMENA, C.A.; FIDALSKI, J. \& ROSSI JÚNIOR, W. Resistência tênsil e friabilidade de um Latossolo sob diferentes sistemas de uso. R. Bras. Ci. Solo, 32:33-42, 2008.
TORMENA, C.A.; SILVA, A.P. \& LIBARDI, P.L. Caracterização do intervalo hídrico ótimo de um Latossolo Roxo sob plantio direto. R. Bras. Ci. Solo, 22:573-581, 1998.

TORMENA, C.A.; SILVA, A.P. \& LIBARDI, P.L. Soil physical quality of a Brazilian Oxisol under two tillage systems using the least limiting water range approach. Soil Till. Res., 52:223-232, 1999.

van GENUCHTEN, M.T. A closed-form equation for predicting the hydraulic conductuvity of unsaturated soils. Soil Sci. Soc. Am. J., 44:892-898, 1980.

VEPRASKAS, M.J. Cone index of loamy sands as influenced by pore size distribution and effective stress. Soil Sci. Soc. Am. J., 48:1220-1225, 1984.

ZOU, C.; SANDS, R.; BUCHAN, G. \& HUDSON, I. Least limiting water range: A potential indicator of physical quality of forest soils. Aust. J. Soil Res., 38:947-958, 2000. 